\title{
G scutem \\ COVID-19 is a risk factor for severe liver damage for patients with intrahepatic cholestasis of pregnancy: a case report
}

\section{Ling Ai}

Department of Anesthesiology, Tongji Hospital, Tongji Medical College, Huazhong University of Science and Technology

\section{Jia Wei}

Department of Obstetrics and Gynecology, Tongji Hospital, Tongji Medical College, Huazhong University of Science and Technology

\section{Shaofang Wang}

Department of Radiology, Tongji Hospital, Tongji Medical College, Huazhong University of Science and Technology

\section{Xi Tan}

Department of Anesthesiology, Tongji Hospital, Tongji Medical College, Huazhong University of Science and Technology

\section{Li Yan}

Department of critical care medicine, Tongji Hospital, Tongji Medical College, Huazhong University of Science and Technology

\section{Shangkun Liu}

Department of Anesthesiology, Tongji Hospital, Tongji Medical College, Huazhong University of Science and Technology

\section{Liu Yang}

School of Management囚Huazhong University of Science and Technology

\section{Ailin Luo}

Department of Anesthesiology, Tongji Hospital, Tongji Medical College, Huazhong University of Science and Technology

Hui Xu ( $\nabla$ huixu@tjh.tjmu.edu.cn )

Department of Anesthesiology, Tongji Hospital, Tongji Medical College, Huazhong University of Science and Technology

\section{Case Report}

Keywords: Intrahepatic cholestasis of pregnancy (ICP), Coronavirus disease 2019 (COVID-19), SARS-CoV2, Liver damage, Case report 
Posted Date: May 4th, 2020

DOI: https://doi.org/10.21203/rs.3.rs-26348/v1

License: (c) (i) This work is licensed under a Creative Commons Attribution 4.0 International License. Read Full License 


\section{Abstract}

Background: Patients with intrahepatic cholestasis of pregnancy (ICP) may present with slight liver damage. In the global outbreak, the number of pregnant women infected with coronavirus disease 2019 (COVID-19) is increasing. For the pregnant patients with ICP, COVID-19 may cause severe liver damage.

Case presentation: A 31-year-old pregnant woman was admitted with fever and respiratory symptoms to Tongji Hospital in Wuhan amid the outbreak of COVID-19. Her chest CT scan showed an infection with viral pneumonia as multiple ground glass opacities in both lungs were spotted. Laboratory tests revealed increased white blood cell (WBC) count and decreased lymphocyte count. The levels of serum total bile acid (TBA) were highly elvated. So were the indices of liver function, including alanine aminotransferase (ALT), aspartate aminotransferase (AST), total bilirubin (TBIL), direct bilirubin (DBIL), alkaline phosphatase (AKP), $\boldsymbol{\gamma}$-glutamyltranspeptidase $(\boldsymbol{\gamma}$-GT), and lactate dehydrogenase (LDH). The patient was later diagnosed of COVID-19 with comorbid ICP, presenting severe liver damage. Through timely termination of pregnancy and effective treatments, the prognoses of the patient and the fetus were well improved.

Conclusions: This case highlights that COVID-19 may be a risk factor of severe liver damage for patients with ICP.Timely termination of pregnancy and effective symptomatic treatments are helpful to improve the progonosis.

\section{Background}

Intrahepatic cholestasis of pregnancy (ICP), is characterized by elevated serum total bile acid (TBA) and/or transaminase concentrations, as well as unexplained pruritus, mostly occurring in the last trimester with spontaneous improvements after delivery. Severe liver damage is rare, and elevations of liver function indexes are generally low, even in severe ICP cases [1].

SARS-CoV-2, the pathogen of coronavirus disease 2019 (COVID-19), has the similar pathological characteristics with severe acute respiratory syndrome coronavirus (SARS-CoV) and Middle East respiratory syndrome coronavirus (MERS-CoV), which had once caused serious pestilence to mankind. The mortality of SARS-CoV infection is $10 \%$, of which the mortality rate of SARS-CoV infection in pregnant women is $25 \%$, and the mortality rate of pregnant women with MERS-CoV infection is up to $37 \%$ $[2,3]$. However, there is only limited data about the clinical features of COVID-19 with pathological pregnancy.

We present a case suffering from both COVID-19 and comorbid ICP who presented with severe liver damage. The patient and the fetus went under a great risk of mortality due to the progressing of the illness. However, due to, correct evaluations, a timely cesarean section and effective further treatments, the patient was successfully delivered and rapidly recovered. 


\section{Case Presentation}

From 12/1/2020, a 31-year-old pregnant woman with gestation of 36 weeks and four days who lived in Jianghan District, Wuhan, considered as an epidemic region of COVID-19, began to experience cough, expectoration, chest tightness and fever. The sputum was little and white, and her highest temperature was $38^{\circ} \mathrm{C}$. At a hospital near her home, she was diagnosed to have an upper respiratory tract infection. Oral medications of Amoxicillin potassium clavulanate and Jinyebaidu granules (Chinese traditional medicine) were prescribed, but she did not show any improvement. On 18/1/2020, the patient began to feel decreased fetal movements, so at $9 \mathrm{pm}$, she was admitted to our emergency department of Tongji Hospital.

The patient had none of comorbid cardiopulmonary diseases, comorbid endocrine diseases, or comorbid infectious diseases before. During pregnancy, the patient presented no symptoms of headache, blurred vision, palpitation or edema. Mild pruritus in the lower extremities appeared from 16/1/2020, and it was tolerable. She had done regular antenatal examinations during pregnancy, and the results of previous visits, including the last one on $10 / 1 / 2020$, showed no abnormalities in obstetric examination and biochemistry.

On admission, she had a fever of $38.5^{\circ} \mathrm{C}$, a heartrate of $98 \mathrm{bpm}$, a respiratory rate of $28 / \mathrm{min}$, and a blood pressure of $134 / 72 \mathrm{mmHg}$. She exhibited a bit of polypnea, with $\mathrm{SpO}_{2} 95 \%$ under no oxygen inhalation. Her breathing in both lungs sounded thick and presented with moist rales. Her fundal height was $32 \mathrm{~cm}$, and her abdominal circumference was $102 \mathrm{~cm}$, with longitudinal lie and cephalic presentation. The fetal heart rate (FHR) was $145 \mathrm{bpm}$ and fetal heart monitoring showed no abnormality.

Laboratory tests on admission showed increased white blood cell (WBC) count $\left(9.86 \times 10^{9} / \mathrm{L}\right)$, increased neutrophil percentage $(86.8 \%)$, decreased lymphocyte count $\left(1.07 \times 10^{9} / \mathrm{L}\right)$, and high TBA $(103.93 \mu \mathrm{mol} / \mathrm{L})$, alanine aminotransferase (ALT) (1574.3U/L), and aspartate aminotransferase (AST) (1593.7 U/L). Some other liver function indexes were elevated as well, such as total bilirubin (TBIL) ( $29.55 \mu \mathrm{mol} / \mathrm{L})$, direct bilirubin (DBIL) $(18.8 \mu \mathrm{mol} / \mathrm{L})$, alkaline phosphatase (AKP) ( $271 \mathrm{U} / \mathrm{L}), \boldsymbol{\gamma}$-glutamyltranspeptidase $(\boldsymbol{\gamma}$-GT) $(303.6 \mathrm{U} / \mathrm{L})$ and lactate dehydrogenase (LDH) $(744 \mathrm{U} / \mathrm{L})$. The level of high sensitive C-creative protein (Hs$\mathrm{CRP})$ rose $(67.28 \mathrm{mg} / \mathrm{L})$ as well. Chest CT showed multiple ground glass opacities, and patchy and strip shadows in both lungs, indicating an infection in both lungs for viral pneumonia, and a small amount of pericardial effusion (Fig.1 A). Considering her typical epidemiological history, symptoms and CT scanning result, detection of SARS-CoV-2 in oropharyngeal swab specimen was sent for examination.

Under the consultation from a MDT, an emergent cesarean section was performed successfully under spinal anesthesia in a designated negative pressure operating room. All personnel involved wore protective equipment according to the third level prevention of infectious diseases, and the patient wore an N95 mask as well. A female infant was delivered with Apgar scores of 8 and 9 at 1 and 5 minutes, and was transferred to neonatology department 10 minutes after birth for close observation and the paitent 
was transferred to the fever ward under 24-hour ECG monitoring and 5L/min oxygen through nasal cannula.

On the first postoperative day (POD), the result of SARS-CoV-2 detection in oropharyngeal swab specimen of the patient was positive, while the detection of the neonate was negative. Laboratory tests were detected again and blood-routine test showed obviously increased WBC count, increased neutrophil count and normal lymphocyte count (Table 1). The levels of ALT and AST were slightly lower compared with the day before, but still obviously higher than the baseline (Table 1). The other indexes of liver function were still highly increased, including AKP, $\boldsymbol{\gamma}-\mathrm{GT}$, LDH, and TBA (Table 1). Inflammatory-related indexes were detected as well, and the results showed the levels of Hs-CRP, erythrocyte sedimentation rate (ESR), procalcitonin (PCT), ferritin, interleukin-2 receptor (IL-2R), interleukin-6 (IL-6), and tumor necrosis factor- $\boldsymbol{\alpha}$ (TNF- $\mathbf{\alpha})$ were increased (Table 1).

Diagnosed as a general case of confirmed COVID-19 with comorbid severe ICP [3], this patient presented severe liver damage. Accordingly, bicyclol (50 mg administered orally every 8 hours) and ursodeoxycholic acid (250mg administered orally every 12 hours) for liver protection and cholagogic, and mucosolvan (90mg administered intravenously every day) for expectorant were given symptomatically. Ganciclovir ( $0.25 \mathrm{~g}$ administered intravenously every 12 hours) for anti-virus was given. Meropenem ( $1 \mathrm{~g}$ administered intravenously every 8 hours) and micafungin sodium ( $0.1 \mathrm{~g}$ administered intravenously every day) were given for anti-bacterial infection empirically. (Fig.2)

After the operation, the patient's vital signs were stable with $\mathrm{SpO}_{2} \geq 98 \%$ under $5-8 \mathrm{~L} /$ min oxygen inhalation through nasal cannula. She had intermittent fever on POD 1 and POD 2, with the highest temperature up to $38.5^{\circ} \mathrm{C}$, and loxoprofen sodium (30 mg administered orally) was given for antipyretic. On POD 3, her body temperature returned to normal, and pruritus disappeared. On POD 7, her cough and expectoration were alleviated. On POD 12, her chest tightness disappeared as well. (Fig.2)

From POD 4, inhalation of recombinant human interferon $\boldsymbol{\alpha} 1 \mathrm{~b}$ (40 $\mu \mathrm{g}$ every 12 hours) for anti-virus was given (Fig.2). From POD 9, cefoperazone sulbactam sodium (3g administered intravenously every 8 hours) was given to replace meropenem (Fig.2).

On POD 12, the patient was tested negative for SARS-CoV-2(Fig.2), and the repeated test on POD 14 remained negative. On POD 12, blood routine tests and inflammatory-related tests returned normal (Table 1). On POD 12, except LDH, most of liver function indexes returned to normal (Table 1). In addition, the test of classification of peripheral blood lymphocytes was normal. Renal function and coagulation tests remained normal as well.

On POD 7 (25/1/2020), chest CT scan was repeated, and showed ground glass opacities in both lungs, indicating viral pneumonia with improvement (Fig.1B). On POD 12 (30/1/2020), the third chest CT scan showed that the shadows were absorbed and well improved, leaving a small amount of pleural effusion (Fig.1C). 
On POD 15, the patient was discharged and sent to the quarantine area for further isolation.

\section{Discussion And Conclusion}

The most sensitive and specific marker for ICP is the elevated level of serum TBA. Patients may present with or without slight liver impairment. Usually, levels of transaminase are slightly elevated, ranging from two folds to 30 -fold of the baseline. And levels of $\boldsymbol{y}-$ GT and LDH are commonly normal.

In addition, limited reports about COVID-19 infection during pregnancy show that the clinical characteristics in pregnant women with confirmed COVID-19 were similar to those for non-pregnant COVID-19 patients in the general population [4,5]. Mild cases and general cases of COVID-19 presented with unobvious liver damage. Among ICU cases, levels of liver function indexes were not significantly increased. In addition, jaundice was rare, only appearing in a few deadly cases [6,7]. In comparison, the patient reported in this case experienced highly increased levels of ALT, AST, AKP, $\boldsymbol{\gamma}-$ GT and LDH, indicating that COVID-19 may be a risk factor of severe liver injuries ICP patients.

Based on the literature and clinical experience, the pathophysiological mechanisms of the patient reported in the case were speculated as follows. First, in addition to alveolar type 2 cells, cholangiocytes also expressed angiotensin converting enzyme2 (ACE2)[8], and could be targeted by SARS-CoV-2 to cause damage to cholangiocytes and hepatocytes. Second, SARS-CoV-2 activated lymphocytes to proliferate and secrete large amounts of pro-inflammatory cytokines, called "cytokine storm", which could attack the tissue cells to cause liver damage, myocardial injury and kidney failure [9]. Third, excessive serum bile acid and toxins caused by ICP in this patient, could accumulate in the liver and helped activate immune cells to magnify "cytokine storm", which accelerated hepatocyte damage [10]. Fourth, contraction of placental blood vessels caused by excessive acid in circulation, could cause damages to the morphology and function of placentas, and induce various oxidative stress responses; hypoxia in hepatocytes was worsened, and the increase of ROS and its peroxides might lead to the release of a variety of pro-inflammatory factors and aggravate ischemia-reperfusion injury (IRI) in liver [11-13].

Delivery decision was made based on both the severity of COVID-19 of the maternal and the gestational age of the fetus. Termination of pregnancy after gestation of 32-34 weeks might be helpful to the followup treatment and prognosis improvement [5]. Monitoring of FHR was important before the delivery, because changes in FHR pattern might serve as an early indicator of maternal respiratory deterioration [14]. In the presented case, considering the great risk to the patient and the fetus, an emergent cesarean section was performed immediately on her admission. After removal of the placenta, hormone levels would return to normal, which might help alleviate the immune imbalance. On the other hand, hypoxia and oxidative stress metabolites produced by contraction of placental blood vessels in ICP could be reduced, and hence, the cytokine storm triggered by SARS-CoV-2 could be alleviated.

At present, antiviral treatment has been routinely used to treat COVID-19 infection in China, but there was little evidence to support the effectiveness of specific antiviral drug. Nebulized $\boldsymbol{\alpha}$-interferon inhalation together with one or two kinds of antiviral drug is recommended in the Chinese guideline. Reduction of 
pulmonary inflammatory exudation caused by bacterial infection could help alleviate "cytokine storm" and organ injuries. In our case, WBC count and neutrophil count were highly elevated on POD 1, meropenem and micafungin sodium were used. Micafungin sodium was empirically used for six days to prevent secondary fungal infection. In case of antibiotic resistance, cefoperazone sulbactam sodium was used to replace eight days treatment of meropenem. Use of corticosteroids in the treatment of COVID-19 pneumonia was not recommended because it might delay the virus clearance. However, methylprednisolone could be used in cases with rapid disease progression or severe illness to improve patients' condition [15].

Bicyclol, a traditional Chinese medicine, has been widely used as a liver protectant when treating with viral hepatitis, fatty liver disease (FLD) and drug induced liver injury(DILI). Bicyclol could maintain hepatocyte membrane stability, alleviate mitochondria injury, decrease nuclear DNA damage and increase the produce of heat shock proteins (HSPs) to stop the progressing of hepatocyte apoptosis [16]. In addition, ursodeoxycholic acid could reduce the concentration of harmful bile acid in the circulation, promote the secretion of bile acid and improve the state of cholestasis. Combined treatment of bicyclol and ursodeoxycholic acid has achieved ideal effects when treating hepatocyte damage complicated with cholestasis.

In our case, the infant was tested negative for SARS-CoV-2 after delivery. This result bears importance regarding the possible maternal-fetus transmission of COVID-19. There have been two cases of COVID19 infection reported by recent literature. One case was confirmed with close contact history. And the other case was confirmed at 36 hours after birth, and close contact could not be ruled out [14]. Despite there is no solid evidence for maternal-fetus transmission, it is recommended that newborns shall be separated from the mother in a timely fashion, and close observation ensue.

Although it is rare, COVID-19 could be a risk factor of severe liver damage for patient with comorbid ICP. Both the patient and the fetus are of great risk of poor outcomes. Early diagnosis, timely termination of pregnancy and appropriate treatments may help improve their prognoses.

\section{Abbreviations}

ACE2: Angiotensin converting enzyme2; AKP: Alkaline phosphatase; ALT: Alanine aminotransferase; AST: Aspartate aminotransferase; COVID-19: Coronavirus disease 2019; DBIL: Direct bilirubin; ESR:

Erythrocyte sedimentation rate; FHR: Fetal heart rate; $\boldsymbol{\gamma}$-GT: $\boldsymbol{\gamma}$-glutamyltranspeptidase; Hs-CRP: High sensitive C-creative protein; ICP: Intrahepatic cholestasis of pregnancy; IL-2R: Interleukin-2 receptor; IL6: Interleukin-6; IRI: Ischemia-reperfusion injury; LDG: Lactate dehydrogenase; MERS-CoV: Middle East respiratory syndrome coronavirus; PCT: Procalcitonin; SARS-CoV: Severe acute respiratory syndrome coronavirus; TBA: Total bile acid; TBIL:Ttotal bilirubin; TNF- $\boldsymbol{\alpha}$ : Tumor necrosis factor- $\boldsymbol{\alpha} ;$ WBC: White blood cell

\section{Declarations}




\section{Ethics approval and consent to participate}

This case report was approved by the Human Assurance Committee (HAC) of Tongji hospital (affiliated with Tongji Medical College, Huazhong University of Science and Technology, Wuhan, China).

\section{Consent for publication}

A written informed consent was obtained from the patient for publishing this case and all the accompanying images. The consent was available for review from the journal editor.

\section{Availability of data and materials}

The datasets of this case were presented within the manuscript. Additional non-sensitive information was available from the corresponding author upon request.

\section{Competing interests}

The authors declare that they have no competing interests.

\section{Funding}

None.

\section{Authors' contributions}

All authors have read and approved the manuscript. LA and HX have full access to all of the data of this case and take responsibility for the integrity and accuracy of the data. Concept and design: SW, XT, Li Y and SL. Acquisition, analysis, or interpretation of data: all authors. Drafting of the manuscript: LA. Critical revision of the manuscript for important intellectual content: JW, Liu Y, AL, and HX.

\section{Acknowledgements}

We thank the patient for granting the permission for publishing the case.

\section{References}

1. Biberoglu E, Kirbas A, Daglar K, Kara O, Karabulut E, Yakut HI, et al. Role of inflammation in intrahepatic cholestasis of pregnancy. J Obstet Gynaecol Res. 2016; 42:252-7.

2. Wong SF, Chow KM, Leung TN, Ng WF, NG TK, Shek CC, et al. Pregnancy and perinatal outcomes of women with severe acute respiratory syndrome. Am J Obstet Gynecol. 2004; 191:292-7.

3. Alserehi H, Wali G, Alshukairi A, Alraddadi B. Impact of Middle East Respiratory Syndrome coronavirus (MERS-CoV) on pregnancy and perinatal outcome. BMC Infect Dis. 2016; 16:105.

4. Qiao J. What are the risks of COVID-19 infection in pregnant women? Lancet. 2020; 395:760-762. 
5. Zhang L, Jiang Y, Wei M, Cheng BH, Zhou XC, Li J, et al. Analysis of the pregnancy outcomes in pregnant women with COVID-19 in Hubei Province. Zhonghua Fu Chan Ke Za Zhi. 2020; 55:E0009.

6. Wang D, Hu B, Hu C, Zhu F, Liu X, Zhang J, et al. Clinical characteristics of 138 hospitalized patients with 2019 novel coronavirus-infected pneumonia in Wuhan, China. JAMA. 2020; doi: 10.1001/jama.2020.1585.

7. Chen N, Zhou M, Dong X, Qu J, Gong F, Han Y, et al. Epidemiological and clinical characteristics of 99 novel coronavirus pneumonia in Wuhan, China: a descriptive study. Lancet. 2020; 395:507-513.

8. Rajapaksha IG, Gunarathne LS, Asadi K, Cunninghan SC, Sharland A, Alexander IE, et al. Livertargeted angiotensin converting enzyme 2 therapy inhibits chronic biliary fibrosis in multiple drugresistant gene 2-knockout mice. Hepatol Commun. 2019; 3:1656-1673.

9. Hu LL, Wang WJ, Zhu QJ, Yang L. Novel coronavirus pneumonia related liver injury: etiological analysis and treatment strategy. Zhonghua Gan Zang Bing Za Zhi. 2020; 28:E001.

10. Woolbright BL, Jaeschke H. Novel insight into mechanisms of cholestatic liver injury. World $\mathrm{J}$ Gastroenterol. 2012; 18:4985-93.

11. Perez MJ, Velasco E, Monte MJ, Gonzalez-Buitrago JM, Marin JJ. Maternal ethanol consumption during pregnancy enhances bile acid-induced oxidative stress and apoptosis in fetal rat liver. Toxicology. 2006; 225:183-94.

12. Zhang XJ, Cheng X, Yan ZZ, Fang J, Wang W, Liu ZY, et al. An ALOX12-12-HETE-GPR31 signaling axis is a key mediator of hepatic ischemia-reperfusion injury. Nat Med. 2018; 24:73-83.

13. Yang L, Wang W, Wang X, Zhao J, Xiao L, Gui W, et al. Creg in hepatocytes ameliorates liver ischemia/reperfusion injury in a TAK1-dependent manner in mice. Hepatology. 2019; 69:294-313.

14. Rasmussen SA, Smulian JC, Lednicky JA, Wen TS, Jamieson DJ. Coronavirus Disease 2019 (COVID19) and pregnancy: what obstetricians need to know. Am J Obstet Gynecol. 2020; doi:10.1016/j.ajog.2020.02.017.

15. Wu C, Chen X, Cai Y, Xia J, Zhou X, Xu S, et al. Risk factors associated with acute respiratory distress syndrome and death in patients with coronavirus disease 2019 pneumonia in Wuhan, China. JAMA Intern Med. 2020; doi: 10.1001/jamainternmed.2020.0994.

16. Liu GT. Bicyclol: a novel drug for treating chronic viral hepatitis B and C. Med Chem. 2009; 5:29-43.

\section{Table}

Table 1. Changes of Laboratory Tests Including Blood-routine, Liver function and Inflammatory Responses 


\begin{tabular}{|c|c|c|c|c|c|c|c|}
\hline Measure & $\begin{array}{c}\text { Reference } \\
\text { Range }\end{array}$ & $\begin{array}{l}\text { Operation } \\
\text { day }\end{array}$ & POD1 & POD3 & POD5 & POD9 & POD12 \\
\hline White blood cell count $\left(\times 10^{9} / \mathrm{L}\right)$ & $3.50-9.50$ & $9.86^{\mathrm{a}}$ & $19.35^{\mathrm{a}}$ & $11.81^{\mathrm{a}}$ & 9.27 & 8.15 & 5.16 \\
\hline Neutrophil percentage (\%) & $40.0-75.0$ & $86.8^{\mathrm{a}}$ & $85^{\mathrm{a}}$ & 74.8 & $77^{\mathrm{a}}$ & $79.2^{\mathrm{a}}$ & 63.5 \\
\hline Neutrophil count $\left(\times 10^{9} / \mathrm{L}\right)$ & $1.80-6.30$ & $8.54^{\mathrm{a}}$ & $16.45^{\mathrm{a}}$ & $8.82^{\mathrm{a}}$ & $7.14^{\mathrm{a}}$ & $8.83^{\mathrm{a}}$ & 3.28 \\
\hline Lymphocyte percentage (\%) & $20.0-50.0$ & $10.8^{\mathrm{b}}$ & $8.3^{\mathrm{b}}$ & $18.1^{\mathrm{b}}$ & $17.2^{\mathrm{b}}$ & 21.2 & 30.6 \\
\hline Lymphocyte count $\left(\times 10^{9} / \mathrm{L}\right)$ & $1.10-3.20$ & $1.07^{\mathrm{b}}$ & 1.61 & 2.14 & 1.59 & 1.37 & 1.58 \\
\hline Eosinophil percentage (\%) & $0.4-8.0$ & $0^{\mathrm{b}}$ & $0^{\mathrm{b}}$ & $0.3^{\mathrm{b}}$ & 1.5 & 1.8 & 1.6 \\
\hline Eosinophil count $\left(\times 10^{9} / \mathrm{L}\right)$ & $0.02-0.52$ & $0^{\mathrm{b}}$ & $0^{\mathrm{b}}$ & 0.04 & 0.14 & 0.22 & 0.08 \\
\hline Alanine aminotransferase (U/L) & $\leq 41$ & $1574.3^{\mathrm{a}}$ & $1382^{\mathrm{a}}$ & $791^{\mathrm{a}}$ & $359^{a}$ & $118^{\mathrm{a}}$ & 30 \\
\hline Aspartate aminotransferase (U/L) & $\leq 40$ & $1593.7^{\mathrm{a}}$ & $1183^{\mathrm{a}}$ & $526^{\mathrm{a}}$ & $70^{\mathrm{a}}$ & $43^{\mathrm{a}}$ & 20 \\
\hline Albumin (g/L) & $35-52$ & $31.1^{\mathrm{b}}$ & $32.9^{\mathrm{b}}$ & $27.6^{\mathrm{b}}$ & $22.4^{\mathrm{b}}$ & $24.1^{\mathrm{b}}$ & $30.3^{\mathrm{b}}$ \\
\hline Total bilirubin ( $\mu \mathrm{mol} / \mathrm{L})$ & $\leq 26$ & 29.55 & 29.9 & $18.9^{\mathrm{b}}$ & $16.2^{\mathrm{b}}$ & $8.2^{\mathrm{b}}$ & $6.8^{\mathrm{b}}$ \\
\hline Direct bilirubin $(\mu \mathrm{mol} / \mathrm{L})$ & $\leq 8.0$ & $18.8^{\mathrm{a}}$ & $28^{\mathrm{a}}$ & $16.7^{\mathrm{a}}$ & $15.6^{\mathrm{a}}$ & 6.2 & 4.1 \\
\hline Alkaline (U/L) & $40-130$ & $271^{\mathrm{a}}$ & $330^{\mathrm{a}}$ & $212^{\mathrm{a}}$ & $160^{\mathrm{a}}$ & $135^{\mathrm{a}}$ & 123 \\
\hline $\boldsymbol{\gamma}$-glutamyltranspeptidase (U/L) & $10-71$ & $303.6^{\mathrm{a}}$ & $327^{\mathrm{a}}$ & $214^{\mathrm{a}}$ & $231^{\mathrm{a}}$ & $147^{\mathrm{a}}$ & $86^{\mathrm{a}}$ \\
\hline Lactate dehydrogenase (U/L) & $135-225$ & $744^{\mathrm{a}}$ & $686^{\mathrm{a}}$ & $562^{\mathrm{a}}$ & $292^{\mathrm{a}}$ & $327^{\mathrm{a}}$ & $306^{\mathrm{a}}$ \\
\hline Total bile acid ( $\mu \mathrm{mol} / \mathrm{L})$ & $\leq 10.00$ & $103.93^{\mathrm{a}}$ & $92.6^{\mathrm{a}}$ & $97.5^{\mathrm{a}}$ & $109.8^{\mathrm{a}}$ & $22^{\mathrm{a}}$ & 7.1 \\
\hline $\begin{array}{l}\text { High sensitive C-creative protein } \\
(\mathrm{mg} / \mathrm{L})\end{array}$ & $<1$ & $67.28^{\mathrm{a}}$ & $44.1^{\mathrm{a}}$ & $38.5^{\mathrm{a}}$ & $21.6^{\mathrm{a}}$ & $11.7^{\mathrm{a}}$ & $5.2^{\mathrm{a}}$ \\
\hline $\begin{array}{l}\text { Erythrocyte sedimentation rate } \\
(\mathrm{mm} / \mathrm{H})\end{array}$ & $0.00-15.00$ & - & $26^{\mathrm{a}}$ & $31^{\mathrm{a}}$ & $16^{\mathrm{a}}$ & 9 & 5 \\
\hline Procalcitonin (ng/ml) & $0.02-0.05$ & - & $0.15^{\mathrm{a}}$ & $0.15^{\mathrm{a}}$ & $0.1^{\mathrm{a}}$ & $0.12^{\mathrm{a}}$ & $0.1^{\mathrm{a}}$ \\
\hline Ferritin $(\mu \mathrm{g} / \mathrm{L})$ & $30-400$ & - & 247.6 & 271.9 & 192.4 & 144.3 & 56.5 \\
\hline Interleukin-1 $\beta$ (pg/ml) & $<5$ & - & $<5$ & $<5$ & $<5$ & $<5$ & $<5$ \\
\hline Interleukin-2 receptor (U/nl) & $223-710$ & - & $1440^{\mathrm{a}}$ & $1468^{\mathrm{a}}$ & $1119^{\mathrm{a}}$ & $875^{\mathrm{a}}$ & 636 \\
\hline Interleukin-6 (pg/ml) & $<7.0$ & - & $82.7^{\mathrm{a}}$ & $77.3^{\mathrm{a}}$ & $69.4^{\mathrm{a}}$ & $17.5^{\mathrm{a}}$ & $6 . .2$ \\
\hline Interleukin-8 (pg/ml) & $<62$ & - & 6.1 & 5.9 & 6.8 & 4.4 & 3.9 \\
\hline Interleukin-10 (pg/ml) & $<9.1$ & - & 8.3 & 7.4 & 3.2 & 7.1 & 5.6 \\
\hline Tumor necrosis factor- $\alpha(\mathrm{pg} / \mathrm{ml})$ & $<8.1$ & - & $13.4^{\mathrm{a}}$ & $25.5^{\mathrm{a}}$ & $17.7^{\mathrm{a}}$ & $9.6^{\mathrm{a}}$ & 4.3 \\
\hline
\end{tabular}

Abbreviations:POD, the day after operation. 


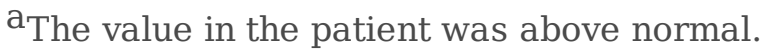

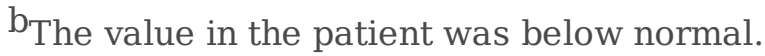

Figures

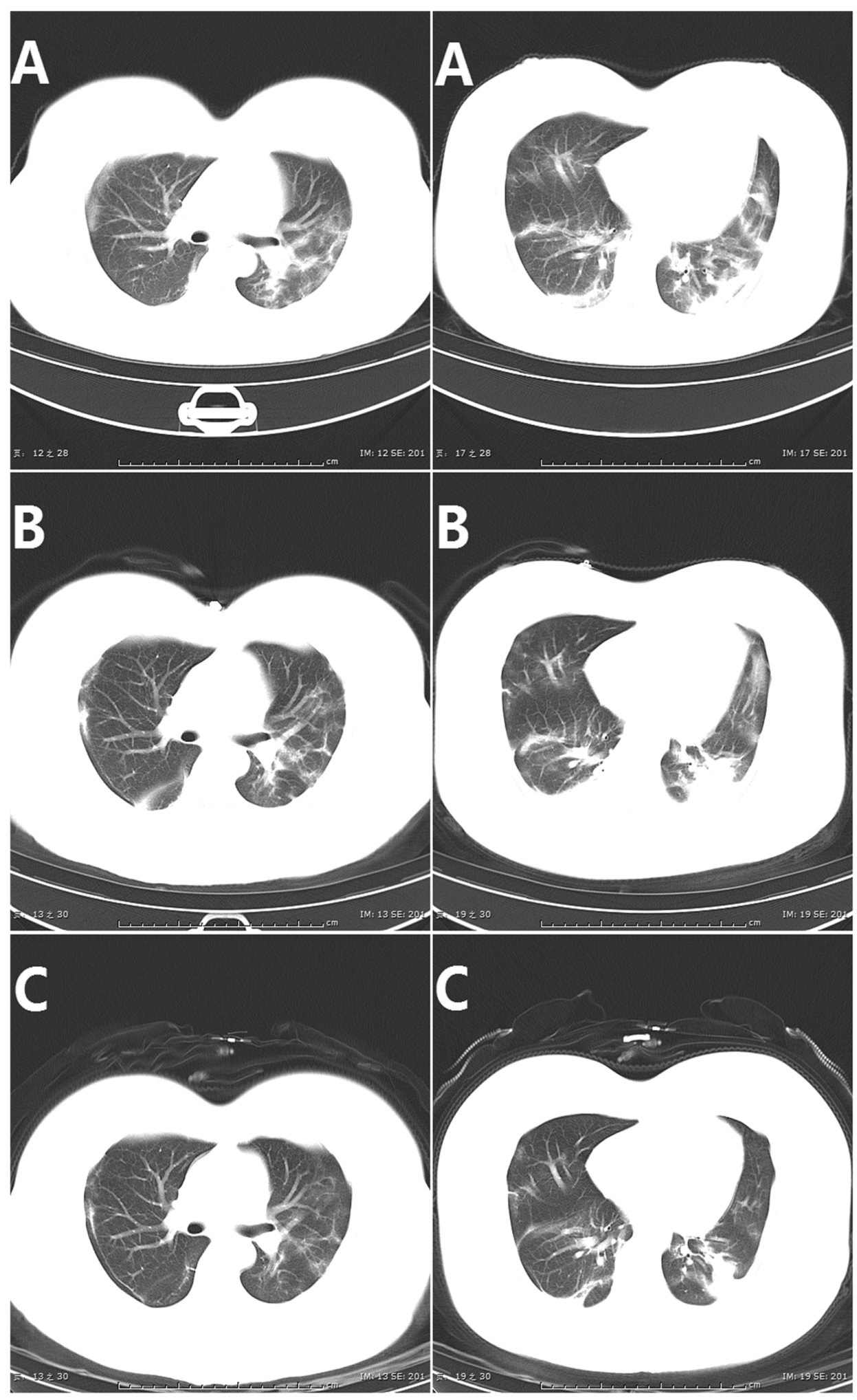

Figure 1 
Chest CT scan of the patient A: Two transverse thin-section chest CT scan on the operation day (18/1/2020). Multiple ground glass opacities, and patchy and strip shadows in both lungs, indicating an infection in both lungs for viral pneumonia, and a small amount of pericardial effusion. B: Two transverse thin-section chest CT scan on POD 7 (25/1/2020). Ground glass opacities in both lungs, indicating viral pneumonia with improvement. C: Two transverse thin-section chest CT scan on POD 12 $(30 / 1 / 2020)$. The shadows were absorbed and well improved, leaving a small amount of pleural effusion.

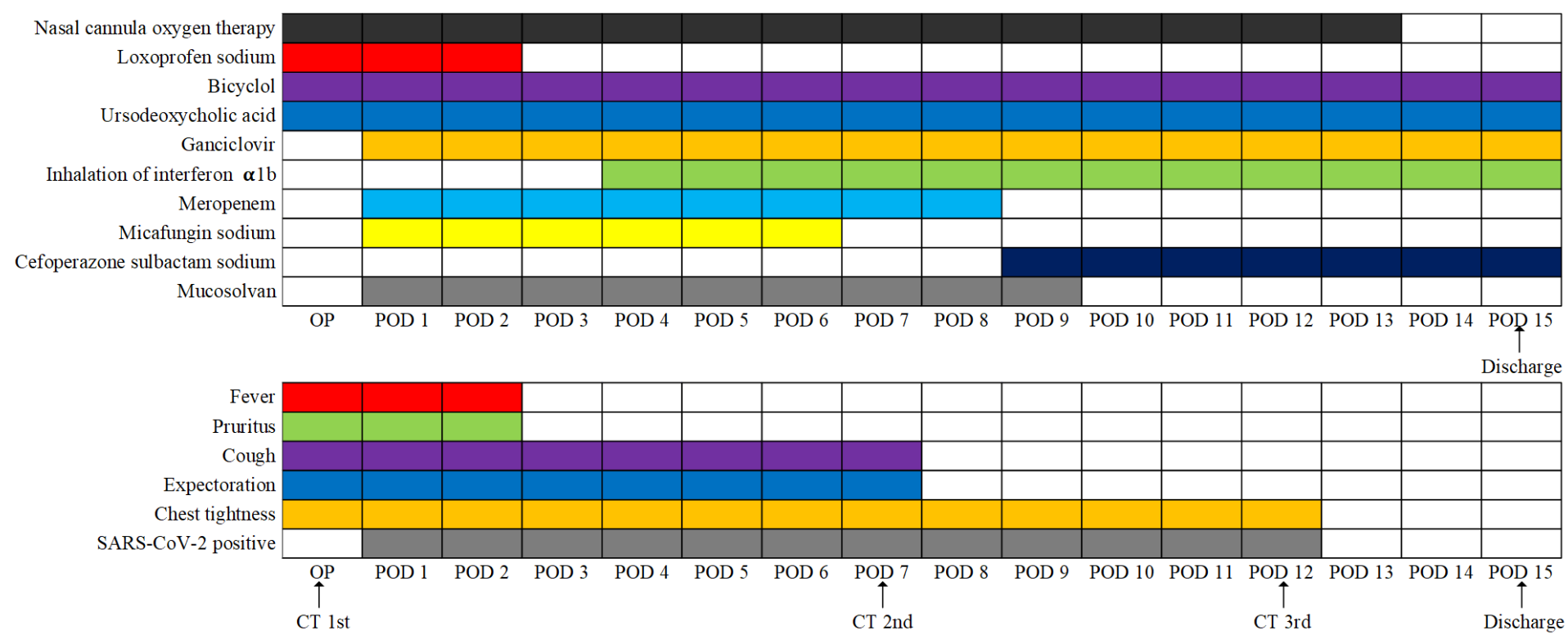

Figure 2

Clinical course of treatments, symptoms and outcomes of the Patient 